\title{
More nonperturbative corrections to the fine and hyperfine splitting in heavy quarkonium
}

\author{
A. Pineda* \\ Departament d'Estructura i Constituents de la Matèria and Institut de Física d'Altes Energies, \\ Universitat de Barcelona, Diagonal, 647, E-08028 Barcelona, Catalonia, Spain \\ (Received 29 May 1996)
}

\begin{abstract}
Leading nonperturbative effects to fine and hyperfine splittings were calculated some time ago. Recently, they have been used in order to obtain realistic numerical results for the lower levels in bottomonium systems. We point out that a contribution of the same order $O\left(\Lambda_{\mathrm{QCD}}^{4} / m^{3} \alpha_{s}^{2}\right)$ has been overlooked. We calculate it in this paper. [S0556-2821(97)02201-7]
\end{abstract}

PACS number(s): 14.40.Gx, 12.38.Lg

\section{INTRODUCTION}

Leading nonperturbative (NP) corrections to fine and hyperfine splittings were calculated a few years ago with the theory developed by Voloshin and Leutwyler [1,2] for heavy quarkonia. Leutwyler computed the hyperfine splitting for $n=1, l=0$ in Ref. [2]. However, a contribution of the same order $O\left(\Lambda_{\mathrm{QCD}}^{4} / m^{3} \alpha_{s}^{2}\right)$ was overlooked. Later on, a number of authors (Krämer et al., Voloshin, and Di Giacomo and co-workers) calculated the fine and hyperfine splittings for different quantum numbers $(n=2,1 ; l=0,1)$ in Ref. [3] where the above overlooked contribution was pointed out, though usually neglected.

Recently, new efforts have been made to obtain realistic numerical results from rigorous QCD in Refs. [4,5] where a coherent picture of bottomonium with $n=1,2$ is provided. Relativistic, radiative, and the leading NP corrections were put together for the first time. Unfortunately, the abovementioned contribution was also omitted.

The aim of this paper is twofold: (i) to complete the calculation up to order $O\left(\Lambda_{\mathrm{QCD}}^{4} / m^{3} \alpha_{s}^{2}\right)$ and (ii) to control the error made by neglecting this contribution. The last point is important if we eventually would like to improve these results by taking into account further orders in the perturbative and NP expansion. We also provide results for a wide range of quantum numbers.

Let us briefly discuss how this contribution arises. Consider the Breit-Fermi interaction in the octet potential

$$
V_{8}^{\text {Coul }} \rightarrow V_{8}^{\text {Coul }}+V_{8}^{\text {BF }}
$$

then, the octet propagator changes into

$$
\frac{1}{E-H_{8}} \rightarrow \frac{1}{E-H_{8}-V_{8}^{\mathrm{BF}}} \simeq \frac{1}{E-H_{8}}+\frac{1}{E-H_{8}} V_{8}^{\mathrm{BF}} \frac{1}{E-H_{8}}
$$

where $H_{8}$ is the octet Coulomb Hamiltonian. This leads to the following correction to the energy:

\footnotetext{
*Electronic address: pineda@ecm.ub.es
}

$$
\begin{aligned}
\delta E_{8}= & -\frac{\pi\left\langle\alpha_{s} G^{2}\right\rangle}{6 N_{c}} \\
& \times\left\langle(n, j, l, s)\left|\vec{r} \frac{1}{E_{n}-H_{8}} V_{8}^{B F} \frac{1}{E_{n}-H_{8}} \vec{r}\right|(n, j, l, s)\right\rangle .
\end{aligned}
$$

$E_{n}$ is the Coulomb singlet bound state energy. $n$ is the principal quantum number. $l, s$, and $j$ are the angular momentum, spin, and total angular momentum, respectively.

Let us comment upon the relative size of this contribution. Notice that it is $1 / N_{c}$ suppressed [in fact $\left(1 /\left(N_{c}^{2}-1\right)\right.$ ] by the ratio of octet $O\left(-1 / 2 N_{c}\right)$ to singlet $O\left(\left(N_{c}^{2}-1\right) / 2 N_{c}\right)$ coupling constant and also it is suppressed by large energy differences between octet and singlet states (recall that the octet potential is repulsive). From this we can conclude that matrix elements with a larger number of octet propagators are numerically suppressed.

Notice also that $V_{8}^{\mathrm{BF}}$ only affects the leading NP results for fine and hyperfine splittings. For the standard NP results found by Leutwyler and Voloshin in Refs. [1,2], Eq. (1.3) gives rise to a subleading contribution which will be neglected in the following. Therefore, we only take into account the piece of $V_{8}^{\mathrm{BF}}$ which contributes to the fine and hyperfine splittings:

$$
V_{8}^{\mathrm{BF}} \doteq V_{8}^{F}+V_{8}^{\mathrm{HF}}=V_{8}^{L S}+V_{8}^{T}+V_{8}^{\mathrm{HF}},
$$

where

$$
\begin{gathered}
V_{8}^{F}=V_{8}^{L S}+V_{8}^{T}=\frac{-1}{2 N_{c}} \frac{3 \alpha_{s}}{2 m^{2}} \frac{1}{r^{3}} \vec{L} \cdot \vec{S}+\frac{-1}{2 N_{c}} \frac{\alpha_{s}}{4 m^{2}} \frac{1}{r^{3}} S_{12}(\hat{r}) \\
S_{12}(\hat{r})=2\left(3 S_{1} \cdot \hat{r} S_{2} \cdot \hat{r}-S_{1} \cdot S_{2}\right) \\
V_{8}^{\mathrm{HF}}=\frac{-1}{2 N_{c}} \frac{4 \pi \alpha_{s}}{3 m^{2}} \delta^{3}(\vec{r}) \vec{S}^{2}
\end{gathered}
$$

and

$$
\delta E_{8} \doteq \delta E_{8}^{F}+\delta E_{8}^{\mathrm{HF}}=\delta E_{8}^{L S}+\delta E_{8}^{T}+\delta E_{8}^{\mathrm{HF}} .
$$


We shall give analytical formulas for the above energies when available.

Let us define the physical fine and hyperfine splittings for $n=2, l=1$. We use spectroscopic notation $2^{2 s+1} P_{j}$. The generalization to arbitrary quantum numbers is straightforward. The fine splittings read

$$
\Delta_{21}=M\left(2{ }^{3} P_{2}\right)-M\left(2{ }^{3} P_{1}\right), \quad \Delta_{10}=M\left(2{ }^{3} P_{1}\right)-M\left(2{ }^{3} P_{0}\right),
$$

while the hyperfine splitting reads

$$
\Delta_{\mathrm{HF}} \equiv \bar{M}\left(2{ }^{3} P\right)-M\left(2{ }^{1} P_{1}\right),
$$

where

$$
\bar{M}\left(2^{3} P\right) \equiv \frac{1}{9}\left[5 M\left(2^{3} P_{2}\right)+3 M\left(2^{3} P_{1}\right)+M\left(2^{3} P_{0}\right)\right]
$$

Typically, $\delta E^{\mathrm{HF}}$ only contributes to $\Delta_{\mathrm{HF}}$ and $\delta E^{F}$ only does to $\Delta_{21}$ and $\Delta_{10}$. Certainly, in our case $\delta E_{8}^{\mathrm{HF}}$ only contributes to $\Delta_{\mathrm{HF}}$ and $\Delta_{21}, \Delta_{10}$ only get contributions from $\delta E_{8}^{F}$, but rather peculiarly, as we will see below, $\Delta_{\mathrm{HF}}$ receives contributions from $\delta E_{8}^{F}$, that is, $\overline{\delta E_{8}^{F}} \neq 0$, where

$$
\begin{aligned}
\overline{\delta E_{8}^{F}}= & \frac{1}{6 l+3}\left\{(2 l+3) \delta E_{8}^{F}(j=l+1)+(2 l+1) \delta E_{8}^{F}(j=l)\right. \\
& \left.+(2 l-1) \delta E_{8}^{F}(j=l-1)\right\}
\end{aligned}
$$

for a general $l$. This is quite unusual and did not happen with the NP corrections calculated previously in the literature where $\overline{\delta E^{F}}=0$.

\section{FINE SPLITTING}

For the fine splitting we obtain

$$
\begin{aligned}
\delta E_{8}^{L S}= & \frac{1}{2 N_{c}} \frac{3 \alpha_{s}}{2 m^{2}} \frac{\pi\left\langle\alpha_{s} G^{2}\right\rangle}{6 N_{c}} \\
& \times \sum_{l_{1}, l_{2}=0}^{\infty} F\left(n, l ; l_{1}, l_{2}\right) G^{L S}\left(j, m, l ; l_{1}, l_{2}\right), \\
\delta E_{8}^{T}= & \frac{1}{2 N_{c}} \frac{\alpha_{s}}{4 m^{2}} \frac{\pi\left\langle\alpha_{s} G^{2}\right\rangle}{6 N_{c}} \\
& \times \sum_{l_{1}, l_{2}=0}^{\infty} F\left(n, l ; l_{1}, l_{2}\right) G^{T}\left(j, m, l ; l_{1}, l_{2}\right) .
\end{aligned}
$$

We have split the matrix elements in radial and angular integrals:

$$
F\left(n, l, l_{1}, l_{2}\right)=\left\langle R_{n l}\left|r \frac{1}{E_{n}-H_{8}^{\left(l_{1}\right)}} \frac{1}{r^{3}} \frac{1}{E_{n}-H_{8}^{\left(l_{2}\right)}} r\right| R_{n l}\right\rangle
$$

TABLE I. We display here $G^{L S}$. For $l=0 j$ must be 1 . The remaining matrix elements are zero.

\begin{tabular}{lccc}
\hline \hline$G^{L S}$ & $j=l-1$ & $j=l$ & $j=l+1$ \\
\hline$l_{1}=l-1$ & $\frac{1-l^{2}}{1+2 l}$ & $\frac{1-l}{1+2 l}$ & $\frac{(-1+l) l}{1+2 l}$ \\
$l_{1}=l$ & 0 & 0 & 0 \\
$l_{1}=l+1$ & $-\frac{(1+l)(2+l)}{1+2 l}$ & $-\frac{2+l}{1+2 l}$ & $\frac{l(2+l)}{1+2 l}$ \\
\hline \hline
\end{tabular}

$$
G^{L S}\left(j, l ; l_{1}, l_{2}\right)=\sum_{j_{1}=\left|l_{1}-1\right|}^{l_{1}+1} \sum_{j_{2}=\left|l_{2}-1\right|}^{l_{2}+1}\langle(j, l, s=1)| \hat{r}_{i} I_{j_{1}, l_{1}}
$$

$$
\times \vec{L} \cdot \vec{S} I_{j_{2}, l_{2}} \hat{r}_{i}|(j, l, s=1)\rangle
$$

$$
\begin{aligned}
G^{T}\left(j, l ; l_{1}, l_{2}\right)= & \sum_{j_{1}=\left|l_{1}-1\right|}^{l_{1}+1} \sum_{j_{2}=\left|l_{2}-1\right|}^{l_{2}+1}\langle(j, l, s=1)| \hat{r}_{i} I_{j_{1}, l_{1}} S_{12}(\hat{r}) \\
& \times I_{j_{2}, l_{2}} \hat{r}_{i}|(j, l, s=1)\rangle
\end{aligned}
$$

where $R_{n l}(r)$ is the radial Coulomb wave function,

$$
\begin{gathered}
|(n, j, l, s)\rangle=\left|R_{n l}\right\rangle|(j, l, s)\rangle, \\
\frac{1}{E_{n}-H_{8}}|l\rangle=\frac{1}{E_{n}-H_{8}^{(l)}}|l\rangle,
\end{gathered}
$$

and $I_{j, l}$ is the identity in subspace with total angular momentum $j$, orbital momentum $l$, and $s=1$ (otherwise the matrix element is 0 ).

Equation (2.3) can be computed using the techniques shown in Ref. [6] but we have not succeeded in finding a close analytical expression for $F\left(n, l ; l_{1}, l_{2}\right)$ although we did succeed for the angular momentum functions. They read

$$
\begin{aligned}
& G^{L S}\left(j, l ; l_{1}, l_{2}\right)=(-1)^{l+l_{1}+1} \delta_{l_{1}, l_{2}}(2 l+1) C\left(l, 1, l_{1} ; 00\right)^{2} \\
& \times \sum_{j_{1}=\left|l_{1}-1\right|}^{l_{1}+1}\left(2 j_{1}+1\right) \\
& \times\left\{\begin{array}{ccc}
l_{1} & j_{1} & 1 \\
j & l & 1
\end{array}\right\}^{2} \\
& \times\left(\frac{j_{1}\left(j_{1}+1\right)-l_{1}\left(l_{1}+1\right)-2}{2}\right) \text {, } \\
& G^{T}\left(j, l ; l_{1}, l_{2}\right)=(2 l+1) C\left(l, 1, l_{1} ; 00\right) C\left(l, 1, l_{2} ; 00\right) \\
& \times \sum_{j_{1}=\left|l_{1}-1\right|}^{l_{1}+1}\left(2 j_{1}+1\right)\left\{\begin{array}{ccc}
l_{1} & j_{1} & 1 \\
j & l & 1
\end{array}\right\} \\
& \times\left\{\begin{array}{ccc}
l_{2} & j_{1} & 1 \\
j & l & 1
\end{array}\right\} \\
& \times 2\left[\delta_{l_{1}, l_{2}}-3 C\left(j_{1}, 1, l_{1} ; 00\right) C\left(j_{1}, 1, l_{2} ; 00\right)\right] \text {. }
\end{aligned}
$$

We display the explicit expressions in Tables I and II. 
TABLE II. We display here $G^{T}$. For $l=0 j$ must be 1 . The remaining matrix elements are either zero or they can be deduced by exchanging $l_{1} \leftrightarrow l_{2}$. The asterisk indicates that the result is only valid for $l \geqslant 2$; otherwise, it is 0 .

\begin{tabular}{lccc}
\hline \hline$G^{T}$ & $l_{1}=l-1, l_{2}=l-1$ & $l_{1}=l-1, l_{2}=l+1$ & $l_{1}=l+1, l_{2}=l+1$ \\
\hline$j=l-1$ & $\frac{2\left(-6-11 l-26 l^{2}-l^{3}+10 l^{4}-12 l^{5}-8 l^{6}\right)}{l(-3+2 l)(-1+2 l)(1+2 l)^{3}}$ & $\frac{-6(1+l)}{(1+2 l)^{3}}$ & $\frac{2(1-2 l)(1+l)(2+l)}{(1+2 l)^{3}}$ \\
$j=l$ & $\frac{2(-1+l)\left(2+3 l+8 l^{2}+4 l^{3}\right)}{l(-1+2 l)(1+2 l)^{2}}$ & $\frac{6}{(1+2 l)^{2}}$ & $\frac{2\left(-6-5 l+7 l^{2}+12 l^{3}+4 l^{4}\right)}{(1+2 l)^{2}\left(3+5 l+2 l^{2}\right)}$ \\
$j=l+1$ & $\frac{2(1-l) l(3+2 l)}{(1+2 l)^{3}}$ & $\frac{-6 l}{(1+2 l)^{3}}$ & $\frac{2\left(-6+14 l+37 l^{2}+l^{3}-50 l^{4}-36 l^{5}-8 l^{6}\right)}{(1+l)(1+2 l)^{3}(3+2 l)(5+2 l)}$ \\
\hline \hline
\end{tabular}

Finally, the final expressions for the energy corrections read

$$
\begin{array}{r}
\delta E_{8}^{L S}=\delta_{s, 1} \frac{\alpha_{s}}{\widetilde{\alpha}_{s}} \frac{\pi\left\langle\alpha_{s} G^{2}\right\rangle}{m^{3}\left(C_{F}{\widetilde{\alpha_{s}}}^{2}\right.} f l s[n, l, j], \\
\delta E_{8}^{T}=\delta_{s, 1} \frac{\alpha_{s}}{\widetilde{\alpha}_{s}} \frac{\pi\left\langle\alpha_{s} G^{2}\right\rangle}{m^{3}\left(C_{F} \widetilde{\alpha_{s}}\right)^{2}} f t[n, l, j] .
\end{array}
$$

$\widetilde{\alpha_{s}}$ was defined in Ref. [4].

We write Eqs. (2.10) and (2.11) in this way in order to make comparison with [5] simpler. We give some numbers for lower values of $n$ :

$$
\begin{aligned}
& (f l s+f t)[1,0,1]=\frac{-9868}{8128125}, \\
& (f l s+f t)[2,0,1]=\frac{-236464}{19780605}, \\
& (f l s+f t)[2,1,2]=\frac{15475732}{415392705}, \\
& (f l s+f t)[3,1,2]=\frac{8134524806}{53682451515}, \\
& (f l s+f t)[2,1,1]=\frac{-452188}{19780605}, \\
& (f l s+f t)[3,1,1]=-\frac{6745478}{59449005}, \\
& (f l s+f t)[2,1,0]=\frac{-1235984}{11868363}, \\
& (f l s+f t)[3,1,0]=-\frac{83789896}{219112047} .
\end{aligned}
$$

In principle our contributions to the fine splitting are quite small in comparison with the NP and radiative corrections coming from the wave functions. Nevertheless, the authors of Ref. [5] managed to isolate the latter in an unknown factor which is determined from the data. Hence, the remaining perturbative and NP effects are kept under control. For the fine splitting they obtain

$$
\Delta_{10}=\frac{5}{4}\left(1+\delta_{\mathrm{rad}}\right) \Delta_{21}-\delta_{\mathrm{NP}}
$$

They take $\Delta_{21}$ from the data, and $\delta_{\text {rad }}$ and $\delta_{\mathrm{NP}}$ are, respectively, the remaining radiative and NP corrections [see (3.2) in the second paper of [5] for details]. While the new contribution reads [to be added to $\delta_{\mathrm{NP}}$ in Eq. (2.13)]

$$
\begin{aligned}
\delta_{\mathrm{NP}}(\text { new }) & =-\frac{5}{4} \delta E_{8}^{F}(j=2)+\frac{9}{4} \delta E_{8}^{F}(j=1)-\delta E_{8}^{F}(j=0) \\
& =-\frac{\alpha_{s}}{\widetilde{\alpha_{s}}} \frac{\pi\left\langle\alpha_{s} G^{2}\right\rangle}{m^{3}\left(C_{F}{\widetilde{\alpha_{s}}}^{2}\right.} \frac{2548892}{415392705}
\end{aligned}
$$

being around $1 \%$ smaller.

\section{HYPERFINE SPLITTING}

In this section we work out the analytical formula for the hyperfine splitting. It receives contributions from both $\overline{\delta E_{8}^{F}}$ and $\delta E_{8}^{\mathrm{HF}}$. Let us calculate $\delta E_{8}^{\mathrm{HF}}$. The angular integral is almost trivial. The $\delta$ function only survives for 0 angular momentum which after composing with $\vec{r}$ implies that only $l=1$ states contribute. It is quite remarkable that only for $l=1$ states do we obtain a nonzero contribution. After these manipulations we find that $\delta E_{8}^{\mathrm{HF}}$ becomes

$$
\begin{aligned}
\delta E_{8}^{\mathrm{HF}}= & s(s+1) \delta_{l, 1} \frac{1}{2 N_{c}} \frac{\alpha_{s}}{9 m^{2}} \frac{\pi\left\langle\alpha_{s} G^{2}\right\rangle}{6 N_{c}} \\
& \times\left|\left\langle R_{n l}\left|r \frac{1}{E_{n}-H_{8}^{\left(l_{1}=0\right)}}\right| r=0\right\rangle\right|^{2} .
\end{aligned}
$$

The radial integral can be carried out by following the formulas given in [6]. We obtain

$$
\begin{gathered}
\delta E_{8}^{\mathrm{HF}}=\delta_{l, 1} s(s+1) \alpha_{s} \frac{\pi\left\langle\alpha_{s} G^{2}\right\rangle}{\left(m{\widetilde{\alpha_{s}}}^{3}\right.} h f[n], \\
h f[n]:=\frac{n^{5}\left(n^{2}-1\right)}{2^{9}} \frac{\Gamma[9 n / 8-2]^{2}}{\Gamma[9 n / 8+3]^{2}} .
\end{gathered}
$$


We again write Eq. (3.2) in this way in order to make comparison with [5] simpler. We give some numbers for lower values of $n(n \geqslant 2)$ :

$$
\begin{gathered}
h f[2]=\frac{65536}{32967675}, \quad h f[3]=\frac{16777216}{296815671075}, \\
h f[4]=\frac{2048}{135270135}, \quad h f[5]=\frac{2097152000}{324918632936187} .
\end{gathered}
$$

Let us now discuss our contribution to $\Delta_{\mathrm{HF}}$. If $l=0$, it turns out to be quite small, but for $l \neq 0$ the leading perturbative order is 0 . Therefore, the next perturbative order is needed and in principle the nonperturbative effects are going to be more important. Thus, in the second paper of [5] a careful analysis of the hyperfine splitting was done for $n=2, l=1$. The following was obtained:

$$
\Delta_{\mathrm{HF}}=\frac{5}{24}\left(\frac{\beta_{0}}{2}-\frac{21}{4}\right) C_{F} \alpha_{s} \Delta_{21}+\frac{976}{1053} \frac{\pi\left\langle\alpha_{s} G^{2}\right\rangle}{m^{3}\left(C_{F} \widetilde{\alpha_{s}}\right)^{2}}
$$

While our contribution reads [to be added to Eq. (3.5)]

$$
\Delta_{\mathrm{HF}}(\text { new })=\overline{\delta E_{8}^{F}}+\delta E_{8}^{\mathrm{HF}}=\frac{\alpha_{s}}{\widetilde{\alpha}_{s}} \frac{\pi\left\langle\alpha_{s} G^{2}\right\rangle}{m^{3}\left(C_{F}{\widetilde{\alpha_{s}}}^{2}\right.} \frac{160277456}{18692671725},
$$

which turns out to be around $1 \%$ smaller.

\section{DISCUSSION}

From the phenomenological point of view our results are going to be important only in the event that a very high precision measurement is done. Nevertheless, they are conceptually important since they take into account the perturbative corrections to the octet Coulomb potential. We have also seen that the hyperfine splitting gets corrections from the fine octet potential which is something somewhat unusual.

Our results complete the calculation of all the contributions of order $O\left(\Lambda_{\mathrm{QCD}}^{4} / m^{3} \alpha_{s}^{2}\right)$. We have also seen the error in neglecting this contribution. This step is unavoidable for an eventual improvement of these results by calculating higher orders in the perturbative and NP expansions.

\section{ACKNOWLEDGMENTS}

I acknowledge a financial support from Generalitat de Catalunya. Financial support from CICYT, Contract No. AEN95-0590, and financial support from CIRIT, Contract No. GRQ93-1047, are also acknowledged. I thank J. Soto for a critical reading of the manuscript and illuminating conversations.
[1] M. B. Voloshin, Nucl. Phys. B154, 365 (1979).

[2] H. Leutwyler, Phys. Lett. 98B, 447 (1981).

[3] A. Krämer, H. G. Dosch, and R. A. Bertlmann, Phys. Lett. B 223, 105 (1989); Fortschr. Phys. 40, 93 (1992); M. B. Voloshin, Sov. J. Nucl. Phys. 35, 592 (1982). M. Campostrini, A. Di Giacomo, and S. Olejnik, Z. Phys. C 31, 577 (1986); G.
Curci, A. Di Giacomo, G. Paffuti, ibid. 18, 135 (1983).

[4] S. Titard and F. J. Ynduráin, Phys. Rev. D 49, 6007 (1994).

[5] S. Titard and F. J. Ynduráin, Phys. Rev. D 51, 6348 (1995); Phys. Lett. B 351, 541 (1995).

[6] M. B. Voloshin, Sov. J. Nucl. Phys. 36, 143 (1982). 